\title{
A Novel Algorithm for Distance Transformation on Irregular Isothetic Grids
}

\author{
Antoine Vacavant ${ }^{1,2}$, David Coeurjolly ${ }^{1,3}$, and Laure Tougne ${ }^{1,2}$ \\ 1 Université de Lyon, CNRS \\ 2 Université Lyon 2, LIRIS, UMR5205, F-69676, France \\ 3 Université Lyon 1, LIRIS, UMR5205, F-69622, France \\ \{antoine.vacavant, david. coeurjolly, laure.tougne\}@liris.cnrs.fr
}

\begin{abstract}
In this article, we propose a new definition of the $\mathrm{E}^{2} \mathrm{DT}$ (Squared Euclidean Distance Transformation) on irregular isothetic grids such as quadtree/octree or run-length encoded $d$-dimensional images. We describe a new separable algorithm to compute this transformation on every grids, which is independent of the background representation. We show that our proposal is able to efficiently handle various kind of classical irregular two-dimensional grids in imagery, and that it can be easily extended to higher dimensions.
\end{abstract}

\section{Introduction}

The representation and the manipulation of large-scale data sets by irregular grids is a today scientific challenge for many applications [9|12. Here, we are interested in discrete distance definition and distance transformation 8813 , on this kind of multi-grids to develop fast and adaptive shape analysis tools. The Distance Transformation (DT) of a binary image consists in labeling each point of a discrete object $\mathcal{E}$ (i.e. foreground) with its shortest distance to the complement of $\mathcal{E}$ (i.e. background). This process is widely studied and developed on regular grids (see for example 44). Some specific extensions of the DT to nonregular grids also exist, such as rectangular grids [15], quadtrees [18, etc. This article deals with generalizing the DT computation on irregular isothetic grids (or $\mathbb{I}$-grid for short) in two dimensions (2-D). The proposed method is easily extensible to higher dimensions. In 2-D, this kind of grids is defined by rectangular cells whose edges are aligned along the two axis. The size and position of those non-overlapping cells are defined without constraint (see next section for a formal definition). The quadtree decomposition and the RLE (Run Length Encoding) grouping schemes are examples of classical techniques in imagery which induce an $\mathbb{I}$-grid. Here, we focus our interest on generalizing techniques that compute the $\mathrm{E}^{2} \mathrm{DT}$ (squared Euclidean DT) of a $d$-dimensional $(d-\mathrm{D})$ binary image 21014. Many of those methodologies can be linked to the computation of a discrete Voronoi diagram of the background pixels 62 . In a previous work [16, we introduced a definition of $\mathrm{E}^{2} \mathrm{DT}$ on $\mathbb{I}$-grids based on the background cells centers. After showing the link between this process and the computation of 
the Voronoi diagram of those background points, we proposed two completing algorithms to handle various configurations of $\mathbb{I}$-grids. Unfortunately, we noticed that they suffer from non-optimal time complexity. Moreover, the result of this transformation strongly depends on the representation of the background.

In this article, we aim to provide a faster version of our previous separable algorithm for distance transformation on $\mathbb{I}$-grids such as quadtree or run-coded $d$-D images. Instead of a center based DT algorithm we now focus on a faster border based DT algorithm, by re-sampling the irregular border part of objects with a partly regular grid. This paper is organized as follows: (1) we present a new way to compute the $\mathrm{E}^{2} \mathrm{DT}$ on $\mathbb{I}$-grids, based on the border of the background cells (Section 2). We also illustrate its relation with the computation of a Voronoi diagram of segments; (2) in Section 3, we describe an algorithm based on the work of R. Maurer et al. [10 to efficiently compute this new transformation in 2-D, but extensible to higher dimensions; (3) we finally show in Section 4 experimental results to compare our new contribution with the techniques we developed in [16] in terms of speed and time complexity.

\section{Distance Transformations on I-grids and Voronoi Diagrams}

In this section, we first recall the concept of irregular isothetic grids (I-grids), with the following definition [1]:

Definition 1 (2-D $\mathbb{I}$-grid). Let $I \subset \mathbb{R}^{2}$ be a closed rectangular support. A 2-D $\mathbb{I}$-grid $\mathbb{I}$ is a tiling of $I$ with non overlapping rectangular cells which edges are parallel to the $X$ and $Y$ axis. The position $\left(x_{R}, y_{R}\right)$ and the size $\left(l_{R}^{x}, l_{R}^{y}\right)$ of a cell $R$ in $\mathbb{I}$ are given without constraint: $\left(x_{R}, y_{R}\right) \in \mathbb{R}^{2},\left(l_{R}^{x}, l_{R}^{y}\right) \in \mathbb{R}^{2}$.

We consider this definition of 2-D I-grids for the rest of the paper, and we will shortly show that our contribution is easily extensible to the $d$-D case. In our framework, we consider labeled I-grids, i.e. each cell of the grid has a foreground or background label (its value is respectively " 0 " or "1" for example). For an $\mathbb{I}$-grid $\mathbb{I}$, we denote by $\mathbb{I}_{F}$ and $\mathbb{I}_{B}$ the sets of foreground and background cells.

We can first consider that the distance between two cells $R$ and $R^{\prime}$ is the distance between their centers. If we denote $p=\left(x_{R}, y_{R}\right)$ and $p^{\prime}=\left(x_{R^{\prime}}, y_{R^{\prime}}\right)$ these points, and $d_{e}^{2}\left(p, p^{\prime}\right)$ the squared Euclidean distance between them, the $\mathbb{I}$-CDT (Center-based DT on $\mathbb{I}$-grids) of a cell $R$ is defined as follows [16]:

$$
\mathbb{I}-\operatorname{CDT}(R)=\min _{R^{\prime}}\left\{d_{e}^{2}\left(p, p^{\prime}\right) ; R^{\prime} \in \mathbb{I}_{B}\right\},
$$

and resumes to the $\mathrm{E}^{2} \mathrm{DT}$ if we consider $\mathbb{I}$ as a regular (square or rectangular) discrete grid. However, this distance is strongly dependent of the background representation. In Figure 11, we present an example of the computation of the $\mathbb{I}$-CDT of two I-grids where only the background cells differ. Since this definition is based on the cells centers position, the I-CDT do not lead to the same distance map depending on the background coding. 


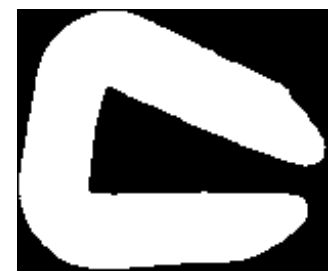

(a)

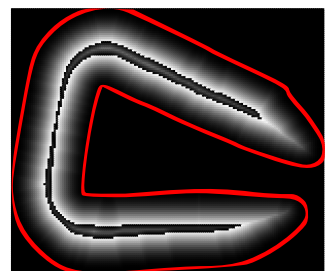

(b)

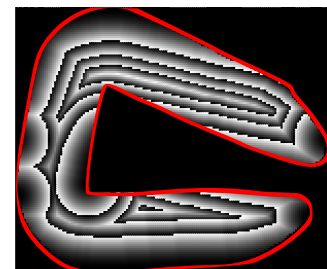

(c)

Fig. 1. The result of the $\mathbb{I}-\mathrm{CDT}$ of the complete regular grid (b) computed from the binary image (a) and an $\mathbb{I}$-grid where the foreground is regular and the background is encoded with a RLE scheme along $Y$ (c). The distance value $d$ of a cell is represented with a grey level $c=d \bmod 255$. The contour of the object (background/foreground frontier) is recalled in each distance map (b) and (c) with a smooth curve.

We now introduce an alternative definition of the $\mathrm{E}^{2} \mathrm{DT}$ by considering the shortest distance between a foreground cell center and the background/foreground boundary of the $\mathbb{I}$-grid. We suppose here that the intersection between two adjacent cells is a segment of dimension one (i.e. they respect the $e$-adjacency relation [1]). Let $\mathcal{S}$ be the set of segments at the interface between two adjacent cells $R \in \mathbb{I}_{F}$ and $R^{\prime} \in \mathbb{I}_{B}$. The $\mathbb{I}$-BDT (Border-based DT on $\mathbb{I}$-grids) is then defined as follows:

$$
\mathbb{I}-\operatorname{BDT}(R)=\min _{s}\left\{d_{e}^{2}(p, s) ; s \in \mathcal{S}\right\} .
$$

Contrary to the $\mathbb{I}-C D T$ given in Equation [1] the result of this process does not depend on the representation of the background. We can draw a parallel between those extensions of the $\mathrm{E}^{2} \mathrm{DT}$ to $\mathbb{I}$-grids and the computation of a Voronoi diagram (VD). More precisely, as in the regular case, the I-CDT can be linked with the VD of the background cells centers (i.e. Voronoi sites or VD sites) [16]. The VD of a set of points $\mathcal{P}=\left\{p_{i}\right\}$ is a tiling of the plane into Voronoi cells (or $V D$ cells $)\left\{C_{p_{i}}\right\}$ [3. If we now consider the background/foreground frontier to compute the $\mathbb{I}$-BDT, our definition implies that we compute a VD of segments, and not a classical VD of points (see Figure 2 for an example of these diagrams computed on a simple $\mathbb{I}$-grid). Hence, a simple approach to compute the I-CDT is to pre-compute the complete VD of the background points [16], and to locate foreground points in the VD. In this case, the $\mathbb{I}$-CDT computation has a $\mathcal{O}\left(n \log n_{B}\right)$ time complexity, where $n$ is the total number of cells, and $n_{B}$ is the number of background cells. This technique is obviously not computationally efficient for every grids, and not adapted to dense grids [16]. To compute the $\mathbb{I}$-BDT, a similar synopsis can be drawn, where the computation of the VD of segments can be handled in $\mathcal{O}\left(n_{S} \log ^{2} n_{S}\right)$, where $n_{S}=4 n_{B}$ is the total number of segments belonging to the background/foreground frontier [7]. The extension of those transformation to $d$-D I-grids is a hard work. A VD can be computed in $d$-D with a $\mathcal{O}\left(n_{B} \log n_{B}+n_{B}^{\lceil d / 2\rceil}\right)$ time complexity (thanks to a gift-wrapping approach [3] for example). However, localizing a point in the VD is an arduous 


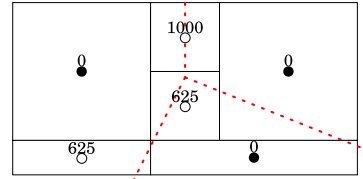

(a)

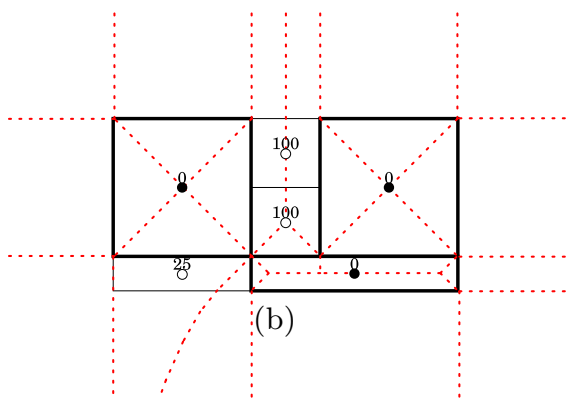

Fig. 2. Example of the VD of the background points to obtain the I-CDT of a simple II-grid (a). Background cell centers are depicted in black, and foreground ones in white. Distance values are presented above the cells centers. From the same grid, the computation of the $\mathbb{I}-\mathrm{BDT}$ (b) implies that we consider the VD of the segments belonging to the background/foreground frontier.

task, and an additional structure like subdivision grids [11] should be constructed to handle this operation.

Hence, we have shown that building the entire VD to obtain the I-BDT is neither computationally efficient for every $\mathbb{I}$-grids, nor easily extensible to higher dimensions. We now propose a separable algorithm to compute the I-BDT that can be extended to the $d$-D case.

\section{A New Separable Algorithm to Compute the I-BDT}

\subsection{R. Maurer et al. $\mathbf{E}^{2}$ DT Algorithm on Regular Grids}

The main idea of this separable (one phase by dimension) method [410] is that the intersection between the complete VD of background pixels (i.e. sites) and a line of the grid can be easily computed, then simplified. Indeed, for a row $j$, the VD sites can be "deleted" by respecting three remarks mainly based on the monotonicity of $d_{e}$ distance [10: (1) if we consider the line $l: y=j, j \in \mathbb{Z}$, then we only have to keep the nearest VD sites from $l$ (Figure 3 b), (2) those sites can be ordered along the $X$ axis, which means that it is not necessary to keep the complete VD, but only its intersection with the line $l,(3)$ a VD site may be hidden by two neighbour sites, and thus not considered anymore (Figure 3-c). In this article, we show the extension of this technique on $\mathbb{I}$-grids by adapting these properties on these grids.

\subsection{Separable Computation of the I-BDT}

To develop a separable process on $\mathbb{I}$-grids, we use a similar structure as the irregular matrix A associated to a labeled $\mathbb{I}$-grid $\mathbb{I}$ introduced in [16. This data structure aims to organize the cells of the grid along the $X$ and $Y$ axis by adding virtual cells centers (see Figure 4 for an example). We used it in our 


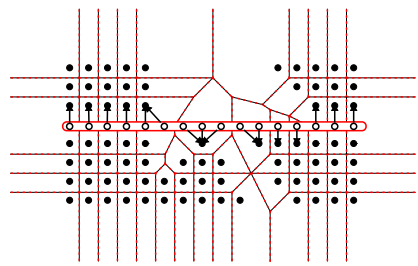

(a)

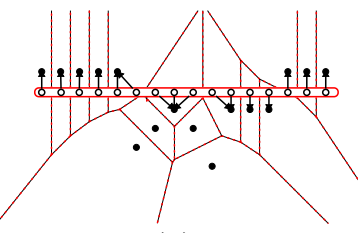

(b)

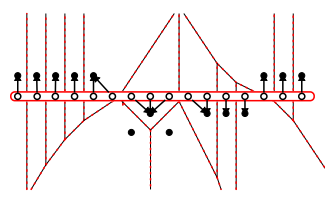

(c)

Fig. 3. In a regular grid, when we treat a row $l$ with R. Maurer et al. algorithm, we consider the VD of background nodes like in (a). We only keep the nearest VD sites of $l$ (b). We obtain (c) by deleting sites which associated VD cells intersect the $l$ row. Arrows indicate the associated VD site of each foreground pixel of this row.

previous work to adapt the separable $\mathrm{E}^{2} \mathrm{DT}$ algorithm of T. Saito et al. [14, devoted to compute the $\mathbb{I}$-CDT on $\mathbb{I}$-grids. The irregular matrix contains as many columns (respectively rows) as $X$-coordinates ( $Y$-coordinates) in the grid. These coordinates are stored in two tables $T_{X}$ and $T_{Y}$ (and we denote $n_{1}=\left|T_{X}\right|$ and $\left.n_{2}=\left|T_{Y}\right|\right)$. At the intersection of two $X$ and $Y$ coordinates, a node in $\mathbf{A}$ has the same value as the cell containing it, and may represent the cell center or not (i.e. this is an extra node, see Figure $4 \mathrm{a}$ ). The extra nodes are used to propagate the distance values through the irregular matrix and then compute a correct distance value for each cell center. To apply the $\mathbb{I}-\mathrm{BDT}$ on this data structure, we also have to take into account the border and the size of the treated cells. We thus add for each node $\mathbf{A}(i, j)$ border attributes along $X$ and $Y$ axis that permit to propagate the distance values to cell borders through $\mathbf{A}$. For a node $\mathbf{A}(i, j)$ contained by the cell $R$ in $\mathbb{I}$, we denote respectively by $H_{L}(i, j)$ and

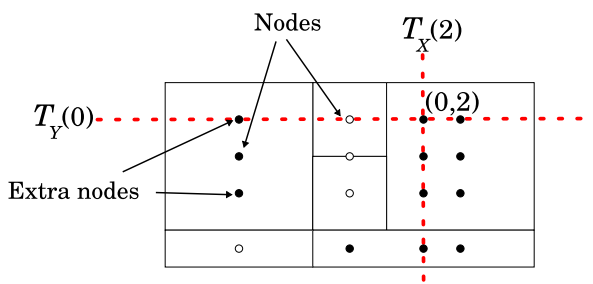

(a)

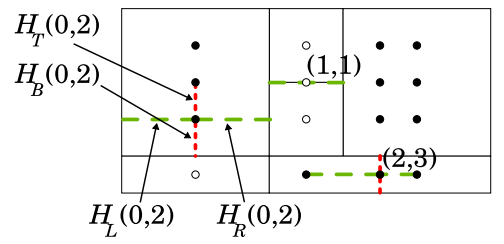

(b)

Fig. 4. Construction of the irregular matrix associated to the simple $\mathbb{I}$-grid illustrated in Figure 2, In (a), the extra node $\mathbf{A}(0,2)$ of the matrix is depicted at the intersection of the dotted lines. New nodes have the same value as the cell containing them. For the I-BDT, we also need the shortest distance between a node in respect to its neighbour nodes and the border of the cell containing it (b). Examples of values for border attributes are also given along $X$ (dashed lines) and $Y$ (dotted lines). For instance, we have $H_{T}(1,1)=H_{B}(1,1)=0$ since this node coincides with a cell horizontal border. We can also notice that $H_{L}(2,3) \neq H_{R}(2,3)$. 
$H_{R}(i, j)$ the attributes that represent the minimum between the distance to the left (respectively right) border of $R$ along $X$, and the distance to the neighbour node at the left (right) position in $\mathbf{A}$. In the same manner, we define $H_{T}(i, j)$ and $H_{B}(i, j)$ in respect to the top (bottom) border of $R$ and neighbour nodes at the top (bottom) position in A (see Figure 4-b). Building the irregular matrix of an $\mathbb{I}$-grid $\mathbb{I}$ can be handled in $\mathcal{O}\left(n_{1} n_{2}\right)$ time complexity. More precisely, we first scan all the cells of $\mathbb{I}$ to know the $n_{1}$ columns and the $n_{2}$ rows of $\mathbf{A}$. Then, we consider each node of $\mathbf{A}$ and we assign its background or foreground value and its border attributes.

Equation 2 can now be adapted on the irregular matrix with a minimization process along the two axis (see [17] for the proof):

Proposition 1 (Separable $\mathbb{I}-\mathbf{B D T}$ ). Let $\mathbf{A}$ be the associated irregular matrix of the 2-D $\mathbb{I}$-grid $\mathbb{I}$. Then the $\mathbb{I}-B D T$ of $\mathbb{I}$ can be decomposed in two separable processes, and consists in computing the matrix $\mathbf{B}$ and $\mathbf{C}$ as follows:

$$
\begin{aligned}
\mathbf{B}(i, j)=\min _{x} & \left\{\min \left(\left|T_{X}(i)-T_{X}(x)-H_{R}(x, j)\right|,\left|T_{X}(x)-T_{X}(i)-H_{L}(x, j)\right|\right) ;\right. \\
\left.x \in\left\{0, \ldots, n_{1}-1\right\}, \mathbf{A}(x, j)=0\right\}, & \mathbf{C}(i, j)=\min _{y}\left\{\mathcal{G}_{y}(j) ; y \in\left\{0, \ldots, n_{2}-1\right\}\right\},
\end{aligned}
$$

where $\mathcal{G}_{y}(j)$ is a flattened parabola given by:

$$
\mathcal{G}_{y}(j)= \begin{cases}\mathbf{B}(i, y)^{2}+\left(T_{Y}(j)-T_{Y}(y)-H_{T}(i, y)\right)^{2} & \text { if } T_{Y}(j)-T_{Y}(y)>H_{T}(i, y) \\ \mathbf{B}(i, y)^{2}+\left(T_{Y}(y)-T_{Y}(j)-H_{B}(i, y)\right)^{2} & \text { if } T_{Y}(y)-T_{Y}(j)>H_{B}(i, y) \\ \mathbf{B}(i, y)^{2} & \text { otherwise. }\end{cases}
$$

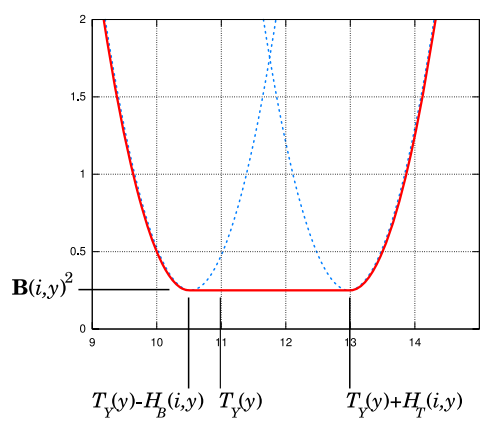

Fig. 5. Important features of a flattened parabola, composition of two half-parabolas (in dotted lines) and a constant function

We prove in 17 that this proposition is correct, i.e. this two-dimensional scheme consists in computing the $\mathbb{I}$-BDT given in Equation 2, This separable minimization process also permits to compute the I-CDT (Equation 11), if we assign all border attributes to zero. In this case, this transformation is equivalent to a minimization process of a 2-D quadratic form [16, and we consider a set of classical 
parabolas along the $Y$ axis, as in the regular case [14. Here, a flattened parabola (see also Figure 5) is an $a d-h o c$ function composed by two half-parabolas and a constant function. It represents a constant distance value from a node $\mathbf{A}(i, j)$ to its neighbour nodes and cell borders, and then increases as a classical quadratic function beyond those limits. The computation of matrix $\mathbf{C}$ in Proposition 1 thus consists in computing the lower envelope of a set of flattened parabolas. This operation is possible since these functions are monotone (composition of monotone functions), and there exist a single intersection or an infinity of intersections between two flattened parabolas. We give in [17 the proof of this property, and the relation between Equation 2 and Proposition 1 .

\subsection{Adaptation of R. Maurer et al. $\mathbf{E}^{2} \mathbf{D T}$ Algorithm on I-grids}

The first stage of our method (first step of Algorithm 1) consists in scanning along $X$ and in initializing the distance of each node of the irregular matrix. This is indeed a double linear scan for each row. Notice the use of $H_{R}$ and $H_{L}$ attributes to propagate the distance to cells borders. At the end of this stage, each node stores a squared distance (line 11). In the second part of our algorithm, we call the Voronoi_IBDT function (Algorithm 2) to build a partial VD intersected with each column $i$. As in the original algorithm [10], we use two stacks storing real successive coordinates of treated sites $(h)$, and their squared distance $(g)$. The first loop of this function (line 2) corresponds to the deletion of hidden sites, thanks to the hidden_by () predicate (Algorithm 3). In Algorithm2, we also use two additional stacks, denoted by $f_{T}$ and $f_{B}$ to store the border attributes and update them (line 22). Thanks to these stacks, the second stage of our algorithm is achieved in linear time. By testing the value of $l$ (line 2),

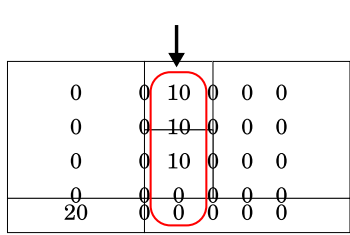

(a)

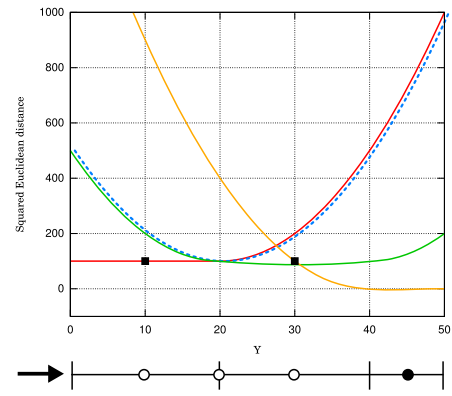

(b)

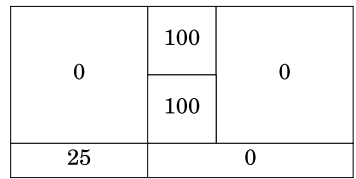

(c)

Fig. 6. For the column chosen in (a), the last phase of Algorithm 2 consists in considering the lower envelope of a set of flattened parabolas (b). At the bottom of this plot, background and foreground nodes are represented by black and white circles at the corresponding $Y$-coordinate. Cell borders are also represented (vertical dashes). Black squares represent where the cell centers are located along $Y$ axis, and the associated $\mathbb{I}$-BDT value. We give in (c) the obtained $\mathbb{I}$-BDT for this $\mathbb{I}$-grid. 

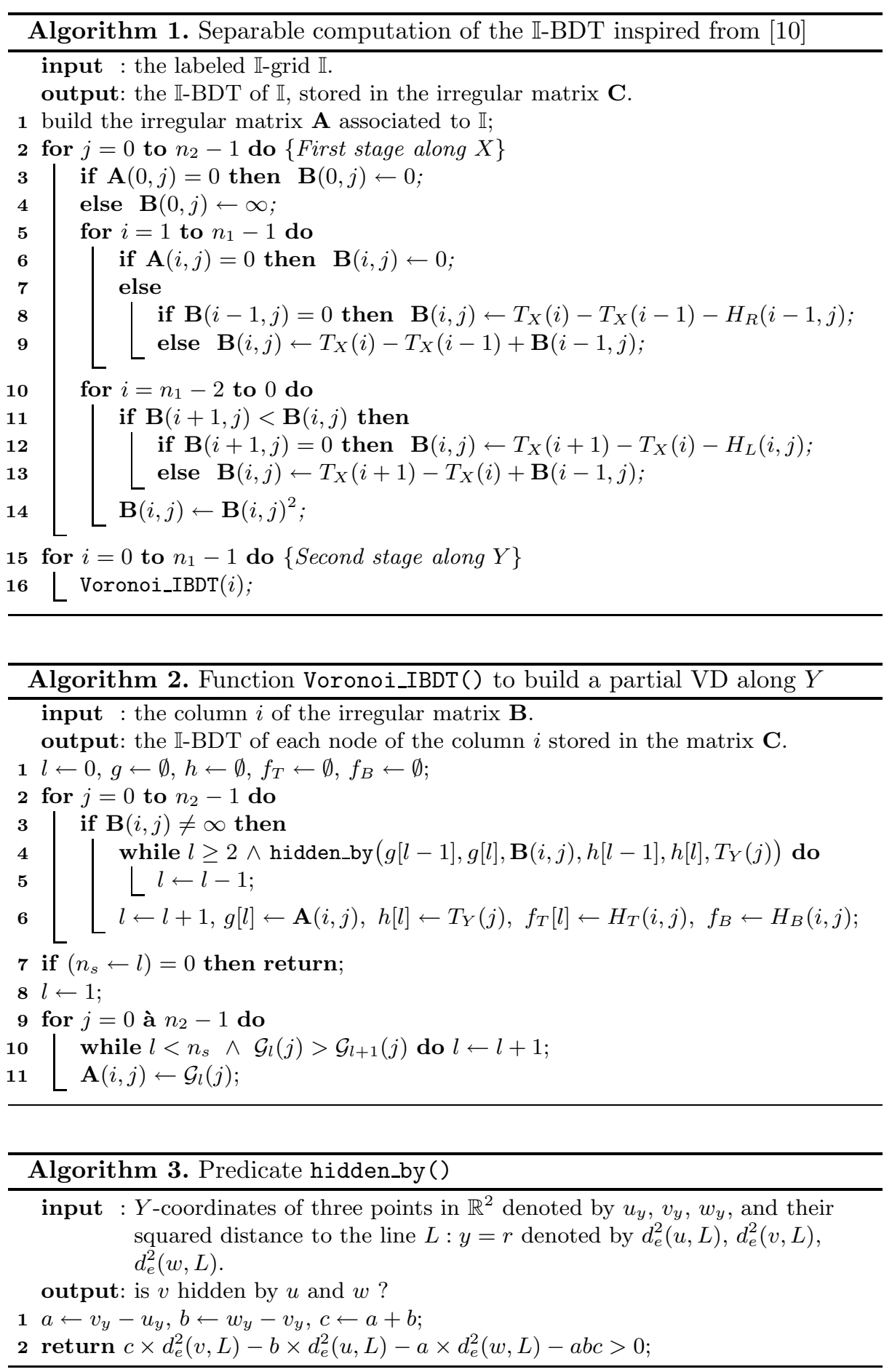
we know if we have to scan again the stacks and to update the distance values of the nodes. Finally, we linearly scan the stacks to find the nearest border of the $\mathbf{A}(i, j)$ current node (line 2), and this step indeed consists in considering the lower envelope of a set of flattened parabolas $\left\{\mathcal{G}_{l}\right\}$, given by (see also Figure 6):

$$
\mathcal{G}_{l}(j)=\left\{\begin{array}{l}
g[l]+\left(T_{Y}(j)-h[l]-f_{T}[l]\right)^{2} \text { if } T_{Y}(j)-h[l]>f_{T}[l] \\
g[l]+\left(h[l]-T_{Y}(j)-f_{B}[l]\right)^{2} \text { if } T_{Y}(j)-h[l]>f_{B}[l] \\
\mathbf{B}(i, y)^{2} \text { else, }
\end{array}\right.
$$

which is the adaptation of Proposition 1 with the stacks $g, h, f_{T}$ and $f_{B}$. We prove in [17] that this adaptation is correct, i.e. it computes a correct distance map for $\mathbb{I}-\mathrm{BDT}$.

As a consequence, we have described a linear $\mathbb{I}$-BDT algorithm in respect to the associated irregular matrix size, i.e. in $\mathcal{O}\left(n_{1} n_{2}\right)$ time complexity. Our new contribution is easily extensible to higher dimensions: we still realize the first step as an initialization phase, and for each dimension $d>1$, we combine results obtained in dimension $d$-1. If we consider a labeled $d$-D II-grid, which associated irregular matrix size is $n_{1} \times \cdots \times n_{d}$, the time complexity of our algorithm is thus in $\mathcal{O}\left(n_{1} \times \cdots \times n_{d}\right)$. In the next section, we present experiments to show the interest of the $\mathbb{I}$-BDT, and to point out that our algorithm is a very efficient approach to compute both $\mathbb{I}$-CDT and $\mathbb{I}$-BDT of various classical $\mathbb{I}$-grids.

\section{Experimental Results}

We first propose to present the result of our I-BDT algorithm for the binary image depicted at the beginning of this article in Figure 1, digitized in various classical $\mathbb{I}$-grids used in imagery. Table 1 illustrates $\mathbb{I}$-BDT elevation maps where the background and the foreground of the original image are independently represented with a regular square grid $(\mathbb{D})$, a quadtree $(q \mathbb{T})$ and a $\operatorname{RLE}$ along $Y$ $(\mathbb{L})$. We can notice in this figure that the result of the $\mathbb{I}$-BDT is independent of the representation of the background. The distance values in the foreground region are thus the same in the three elevation maps of a given column.

We now focus our interest on the execution time of our new algorithm, in respect to our previous work [16. We consider the three algorithms presented in Table 2. The first algorithm represents the simple approach we discussed in Section 2, which is hardly extensible to $d$-D treatments, and I-BDT computation. Algorithm 2 is described in [16] and is inspired from the quadratic form minimization scheme of T. Saito et al. [14. Thanks to the flattened parabola definition, we can extend this algorithm to I-BDT, but with a non-optimal time complexity, in respect to the irregular matrix size. Algorithm 3 is our new separable transformation inspired from [10. In Figure 7], we present the three chosen images for our experiments, and in Table 3, we show the execution times for these three algorithms, for $\mathbb{I}$-grids built from three binary images. We have performed those experiments on a mobile workstation with a $2.2 \mathrm{Ghz}$ Intel Centrino Duo processor, and $2 \mathrm{~Gb}$ RAM. We can first notice that our new contribution gives good results for $\mathbb{I}$-CDT. Indeed, Algorithm 3 is the fastest one for dense 


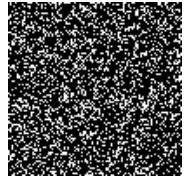

(a) noise

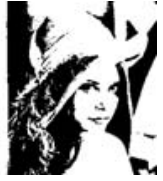

(b) lena

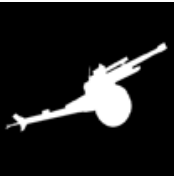

(c) canon

Fig. 7. For the images noise (100x100), lena (208x220), canon (512x512) we consider in our experiments the associated $\mathbb{I}$-grids (regular grid, quadtree decomposition and RLE along $Y$ scheme)

Table 1. Elevation maps representing the $\mathbb{I}$-BDT of each $\mathbb{I}$-grid. $X$ and $Y$ are the axis of the image, and $Z$ corresponds to the distance value. The foreground (columns) and the background (rows) of the image are digitized independently. The color palette is depicted on the right of the figure.

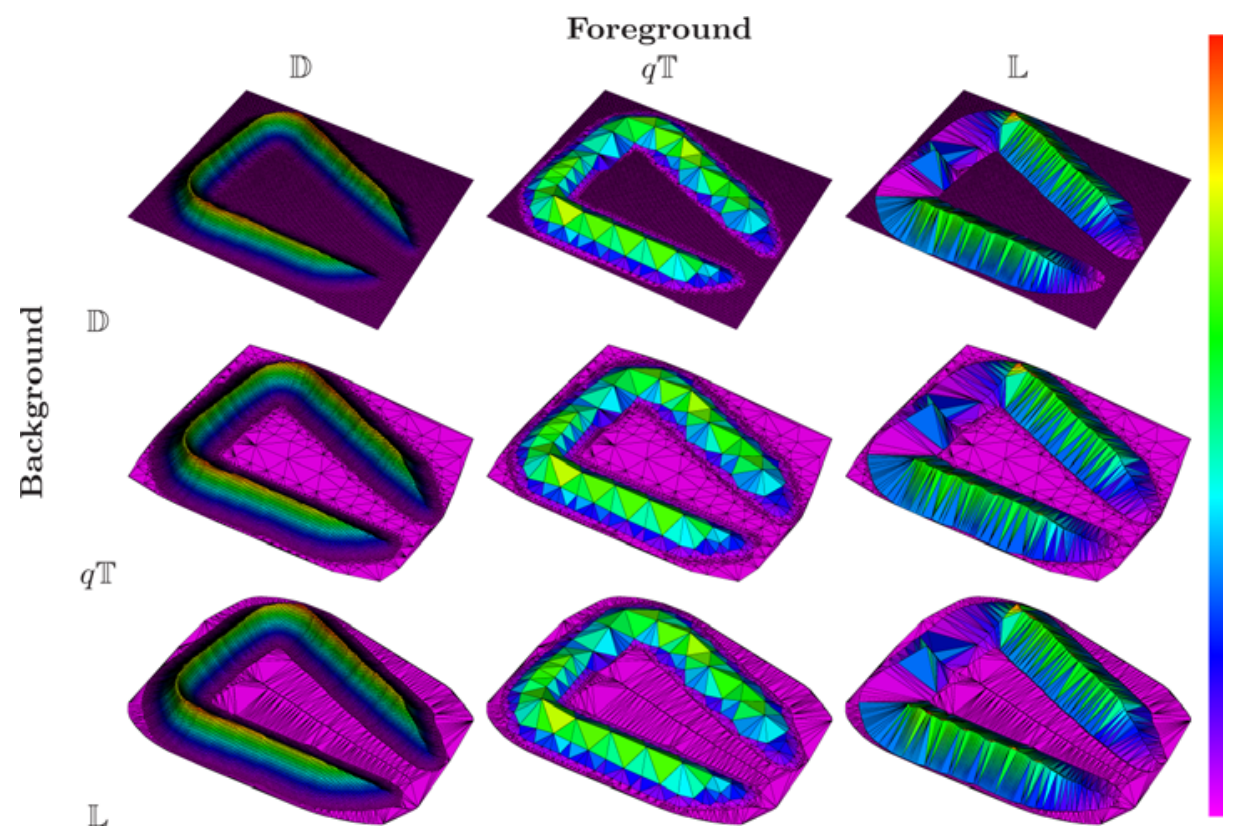

Table 2. The three compared algorithms, and their associated time and space complexities. We also check if an algorithm is extensible to $d$-D $\mathbb{I}$-grids and what kind of transformation it can perform (I-CDT, $\mathbb{I}$-BDT).

\begin{tabular}{|c|c|c|c|c|c|}
\hline & Algorithm & Time & Space & $d$-D $\mathbb{I}-\mathbf{C D T}$ & $\overline{\mathbb{I}-\mathrm{BDT}}$ \\
\hline 1 & Complete VD [16] & $\overline{\mathcal{O}\left(n \log n_{B}\right)}$ & $\mathcal{O}(n)$ & $\sqrt{ }$ & \\
\hline 2 & From T. Saito et al. 1416 & $\mathcal{O}\left(n_{1} n_{2} \log n_{2}\right)$ & $\mathcal{O}\left(n_{1} n_{2}\right)$ & $\checkmark$ & \\
\hline 3 & From R. Maurer et al. 10 & $\mathcal{O}\left(n_{1} n_{2}\right)$ & $\mathcal{O}\left(n_{1} n_{2}\right)$ & $\checkmark$ & $\checkmark$ \\
\hline
\end{tabular}


Table 3. We present execution times (in seconds) for each algorithm for the I-CDT (a) and the $\mathbb{I}$-BDT (b) and for each $\mathbb{I}$-grid. Number inside parenthesis in (b) are the increasing rate in $\%$ between $\mathbb{I}$-CDT and $\mathbb{I}$-BDT execution times.

(a) $\mathbb{I}$-CDT

\begin{tabular}{|c|c|c|c|c|c|c|c|c|c|}
\hline \multirow{2}{*}{ Image } & \multicolumn{3}{|c|}{ Algorithm 1} & \multirow{2}{*}{ Image } & \multicolumn{3}{|c|}{ Algorithm 2} & \multirow{2}{*}{ Image } & Algorithm 3 \\
\hline & $\mathbb{D}$ & $q \mathbb{T}$ & $\mathbb{L}$ & & $\mathbb{D}$ & $q \mathbb{T}$ & $\mathbb{L}$ & & $q \mathbb{T}$ \\
\hline & 0.255 & 0.104 & - & & 0.037 & $\pi$ & $\overline{.044}$ & & 0.04 \\
\hline & 1.413 & & & & 0.1 & & & & 0. \\
\hline canon & 36.236 & 0.27 & 0.234 & canon & 1.678 & 1.322 & 1.316 & canon & 1.1340 .4850 .585 \\
\hline
\end{tabular}

(b) $\mathbb{I}-\mathrm{BDT}$

\begin{tabular}{cccccccccc}
\hline Image & \multicolumn{3}{c}{ Algorithm 2 } & & Image & \multicolumn{3}{c}{ Algorithm 3 } \\
& $\mathbb{D}$ & $q \mathbb{T}$ & $\mathbb{L}$ & & & $\mathbb{D}$ & $q \mathbb{T}$ & $\mathbb{L}$ \\
\hline noise & $0.047(27)$ & $0.100(29)$ & $0.054(23)$ & & noise & $0.065(42)$ & $0.085(31)$ & $0.049(29)$ \\
lena & $0.256(33)$ & $0.491(31)$ & $0.320(31)$ & & lena & $0.258(40)$ & $0.209(26)$ & $0.170(26)$ \\
canon & $2.248(34)$ & $2.107(59)$ & $2.020(53)$ & & canon & $1.507(33)$ & $0.563(16)$ & $0.718(23)$ \\
\hline
\end{tabular}

$\mathbb{I}$-grids (regular grids or $\mathbb{L}$ for noise), and is very competitive for sparse $\mathbb{I}$-grids (e.g. near one half second for $q \mathbb{T}$ and $\mathbb{L}$ based on image canon). In the latter case (canon), Algorithm 1 is slightly faster than our approach, but we recall that it is hardly extensible to higher dimensions. Algorithm 2, which we developed in our previous work [16], was interesting for dense grids, and is now overtaken by Algorithm 3. For the I-BDT, Algorithm 2 and 3 suffer from an execution time increase, mainly due to the integration of the complex flattened parabola. But our contribution remains as the fastest algorithm, and the time increasing rate is moderate for the tested grids. Large sparse $\mathbb{I}$-grids like $q \mathbb{T}$ and $\mathbb{L}$ based on canon are still handled in less than one second.

\section{Conclusion and Future Works}

In this article, we have proposed a new extension of the $\mathrm{E}^{2} \mathrm{DT}$ on $\mathbb{I}$-grids based on the background/foreground frontier, and independent of the background representation. We have also developed a new separable algorithm inspired from [10] that is able to efficiently compute the I-BDT thanks to the irregular matrix structure. We have finally shown that our new contribution is adaptable to various configurations of $\mathbb{I}$-grids, with competitive execution time and complexities, in comparison with our previous proposals.

As a future work, we would like to develop the extension of the discrete medial axis transform [2] on $\mathbb{I}$-grids with our new definition. This would permit to propose a centred simple form of a binary object, independently of the representation of the background, and surely extensible to higher dimensions. As in this paper, we aim to propose a linear algorithm to construct an irregular medial axis, in respect to the irregular matrix size. 


\section{References}

1. Coeurjolly, D., Zerarga, L.: Supercover Model, Digital Straight Line Recognition and Curve Reconstruction on the Irregular Isothetic Grids. Computer and Graphics 30(1), 46-53 (2006)

2. Coeurjolly, D., Montanvert, A.: Optimal Separable Algorithms to Compute the Reverse Euclidean Distance Transformation and Discrete Medial Axis in Arbitrary Dimension. IEEE Transactions on Pattern Analysis and Machine Intelligence 29(3), 437-448 (2007)

3. de Berg, M., van Kreveld, M., Overmars, M., Schwarzkopf, O.: Computational Geometry: Algorithms and Applications. Springer, Heidelberg (2000)

4. Fabbri, R., Costa, L.D.F., Torelli, J.C., Bruno, O.M.: 2D Euclidean Distance Transform Algorithms: A Comparative Survey. ACM Computing surveys 40(1), 1-44 (2008)

5. Fouard, C., Malandain, G.: 3-D Chamfer Distances and Norms in Anisotropic Grids. Image and Vision Computing 23(2), 143-158 (2005)

6. Hesselink, W.H., Visser, M., Roerdink, J.B.T.M.: Euclidean Skeletons of 3D Data Sets in Linear Time by the Integer Medial Axis Transform. In: 7th International Symposium on Mathematical Morphology, pp. 259-268 (2005)

7. Karavelas, M.I.: Voronoi diagrams in CGAL. In: 22nd European Workshop on Computational Geometry (EWCG 2006), pp. 229-232 (2006)

8. Klette, R., Rosenfeld, A.: Digital Geometry: Geometric Methods for Digital Picture Analysis. Morgan Kaufmann Publishers Inc., San Francisco (2004)

9. Knoll, A.: A Survey of Octree Volume Rendering Techniques. In: 1st International Research Training Group Workshop (2006)

10. Maurer, C.R., Qi, R., Raghavan, V.: A Linear Time Algorithm for Computing Exact Euclidean Distance Transforms of Binary Images in Arbitrary Dimensions. IEEE Transactions on Pattern Analysis and Machine Intelligence 25(2), 265-270 (2003)

11. Park, S.H., Lee, S.S., Kim, J.H.: The Delaunay Triangulation by Grid Subdivision. Computational Science and Its Applications, 1033-1042 (2005)

12. Plewa, T., Linde, T., Weirs, V. (eds.): Adaptive Mesh Refinement - Theory and Applications. In: Proceedings of the Chicago Workshop on Adaptive Mesh Refinement in Computational Science and Engineering, vol. 41 (2005)

13. Rosenfeld, A., Pfalz, J.L.: Distance Functions on Digital Pictures. Pattern Recognition 1, 33-61 (1968)

14. Saito, T., Toriwaki, J.: New Algorithms for n-dimensional Euclidean Distance Transformation. Pattern Recognition 27(11), 1551-1565 (1994)

15. Sintorn, I.M., Borgefors, G.: Weighted Distance Transforms for Volume Images Digitized in Elongated Voxel Grids. Pattern Recognition Letters 25(5), 571-580 (2004)

16. Vacavant, A., Coeurjolly, D., Tougne, L.: Distance Transformation on TwoDimensional Irregular Isothetic Grids. In: Coeurjolly, D., Sivignon, I., Tougne, L., Dupont, F. (eds.) DGCI 2008. LNCS, vol. 4992, pp. 238-249. Springer, Heidelberg (2008)

17. Vacavant, A., Coeurjolly, D., Tougne, L.: A Novel Algorithm for Distance Transformation on Irregular Isothetic Grids. Technical report RR-LIRIS-2009-009 (2009), http://liris.cnrs.fr/publis/?id=3849

18. Vörös, J.: Low-Cost Implementation of Distance Maps for Path Planning Using Matrix Quadtrees and Octrees. Robotics and Computer-Integrated Manufacturing 17(6), 447-459 (2001) 